\title{
Attosecond polarization modulation of $x$-ray radiation in a free-electron laser
}

\author{
J. Morgan $\odot^{*}$ \\ University of Strathclyde (SUPA), Glasgow G4 ONG, United Kingdom \\ and Cockcroft Institute, Warrington, WA4 4AD, United Kingdom \\ B. W. J. McNeilø \\ University of Strathclyde (SUPA), Glasgow G4 ONG, United Kingdom; \\ Cockcroft Institute, Warrington, WA4 4AD, United Kingdom; \\ and ASTeC, STFC Daresbury Laboratory, Warrington, WA4 4AD, United Kingdom
}

(Received 18 August 2020; accepted 7 January 2021; published 14 January 2021)

\begin{abstract}
A new method to generate short wavelength free-electron laser output with modulated polarization at attosecond timescales is presented. Simulations demonstrate polarization switching timescales that are four orders of magnitude faster than the current state of the art and, at x-ray wavelengths, approaching the atomic unit of time of approximately 24 attoseconds. Such polarization control has significant potential in the study of ultrafast atomic and molecular processes. The output alternates between either orthogonal linear or circularly polarized light without the need for any polarizing optical elements. This facilitates operation at the high brightness x-ray wavelengths associated with FELs. As the method uses an afterburner configuration it would be relatively easy to install at existing FEL facilities, greatly expanding their research capability.
\end{abstract}

DOI: 10.1103/PhysRevAccelBeams.24.010701

\section{INTRODUCTION}

The polarization of light is a fundamental property which affects its interactions with matter. These interactions are used experimentally to investigate various properties of matter such as the chirality of molecules and crystal structures [1,2]. Additionally, light's polarization is essential in the study of magnetism, for example, short polarized pulses can be used to manipulate magnetic moments and investigate the timescales of angular momentum exchange [3]. Current experiments can demand greater spatial [4] and/or temporal [5] control and flexibility of the polarization than the generation of purely circular, elliptical or linearly polarized light. In particular, fast temporal switching of light's polarization is desirable for techniques such as polarization modulation spectroscopy, notably, x-ray magnetic circular dichroism (XMCD) [6-8] and may offer control over material excitations including lattice vibrations [9], charge and spin [10,11].

Ultrafast switching of the polarization properties of light is a nontrivial task as conventional polarizing elements are

jenny.morgan@strath.ac.uk

Published by the American Physical Society under the terms of the Creative Commons Attribution 4.0 International license. Further distribution of this work must maintain attribution to the author(s) and the published article's title, journal citation, and DOI. quasistatic devices at ultra-fast timescales. While some conventional polarizing elements can be controlled by electric currents [12], these are limited by their electronic components to gigahertz switching speeds and also see large energy losses.

In the drive to further decrease switching rates, recent research using plasmonic technologies has further decreased linear polarization switching to 800 fs [13] and circular polarization switching to pico-second timescales [14]. However, these techniques are based on the active control of polarizing elements and operate primarily at visible wavelengths or longer. As wavelengths shorten beyond the ultraviolet, polarizing optics are more limited with modulation timescales being determined primarily by the light generation method.

In electron accelerator based light sources, which can generate light into the hard x-ray, it is the motion of the radiating electrons propagating through magnetic undulators that determines the polarization of the photon beam. For example, circular polarization modulation with a $\sim 2 \mathrm{~ns}$ switching rate has been demonstrated in a synchrotron by controlling electron bunch orbits through twin undulators [15]. Methods of generating x-rays with temporally varying polarization from the output of free-electron lasers, FELs, include $100 \mathrm{fs}$ switching using a chirped electron beam [16]. Femto-second isolated pulses with different polarization can be generated with the fresh slice method described in [17]. 
It can be envisaged that significant improvement on temporal polarization switching timescales to those comparable to atomic processes will enable experimental investigation of these processes. In XMCD, for example, the polarization switching rate of $\mathrm{x}$-rays limits the observable processes to those occurring slower than the switching cycle [8]. In this paper, a method is described which could improve the polarization switching rate of both linear and circularly polarized high brightness $\mathrm{x}$-rays toward the attosecond timescale regime and comparable to the period of a ground state electron in the Bohr hydrogen atom, the atomic unit of time $\approx 24$ as. We speculate that pulses of such timescales could be used to develop novel methods, perhaps similar to $\mathrm{XMCD}$, that can improve temporal resolutions to below that of the exchange interaction responsible for magnetic order [3].

The method modulates the polarization of light radiated from a free-electron laser. Trains of radiation pulses are generated in which each pulse alternates between orthogonal linear or circular polarization states. FELs are widely tuneable devices operating down to the hard $\mathrm{x}$-ray wavelength range [18] and the method reported here should be applicable over this full range. Simulations are carried out here in the soft $\mathrm{x}$-ray generating radiation pulse trains with alternate orthogonal polarization pulse timescales of tens of attoseconds.

\section{FEL THEORY}

In a high-gain FEL a relativistic electron beam propagates through magnetic undulators and emits electromagnetic radiation (light) with a resonant wavelength $\lambda_{r}=\lambda_{u}\left(1+\bar{a}_{u}^{2}\right) / 2 \gamma_{0}^{2}$, where $\lambda_{u}$ is the undulator period, $\bar{a}_{u}$ is the rms undulator parameter and $\gamma_{0}$ is the electron beam's relativistic factor. The light is amplified via a collective interaction which causes the electrons to micro-bunch at the resonant wavelength and to emit coherently [18]. The initial nonuniform phase distribution of electrons, or shot-noise, can provide the initial seed which is subsequently amplified in the process of self-amplified spontaneous emission, giving a temporally noisy output [19]. The relative propagation of a radiation wavefront through the electrons of one $\lambda_{r}$ every $\lambda_{u}$, referred to as "slippage," allows interaction between different regions of the electron bunch and radiation pulse. This correlates the phase of the radiation output at a length determined by the cooperation length $l_{c}=\lambda_{r} / 4 \pi \rho$ - the relative slippage in an exponential gain length through the undulator $l_{g}=\lambda_{u} / 4 \pi \rho$. Here $\rho$, the FEL parameter, determines the strength of the FEL interaction [19].

The FEL process generates high-power radiation with its polarization determined by the magnetic undulator fieldeither planar, elliptical or helical. A typical X-ray FEL facility uses planar undulators to micro-bunch the electrons. Polarization control can be enabled by adding additional undulators placed downstream of the main planar undulator amplification section once microbunching has been established [20]. Such additional downstream undulators, or "afterburners," are increasingly being explored as a method to tailor FEL output in many ways not limited to polarization control, e.g., short pulse generation [21,22] and transverse phase manipulation [23]. They provide solutions to enable specific experimental output requirements with minimal changes to an existing facility and therefore at relatively low cost.

\section{POLARIZATION MODULATED PULSE TRAINS: METHOD}

To generate FEL output with modulated polarization, we propose an afterburner design consisting of a series of few period, alternate orthogonally polarized undulator modules as shown in Fig. 1. The undulators are separated by electron delay chicanes which can introduce additional slippage between the electron bunch and the radiation field. Both of the orthogonal, polarized radiation fields emitted in the afterburner are mode-locked which creates trains of short pulses [21]. The orthogonally polarized pulse trains are shifted temporally with respect to each other so that the

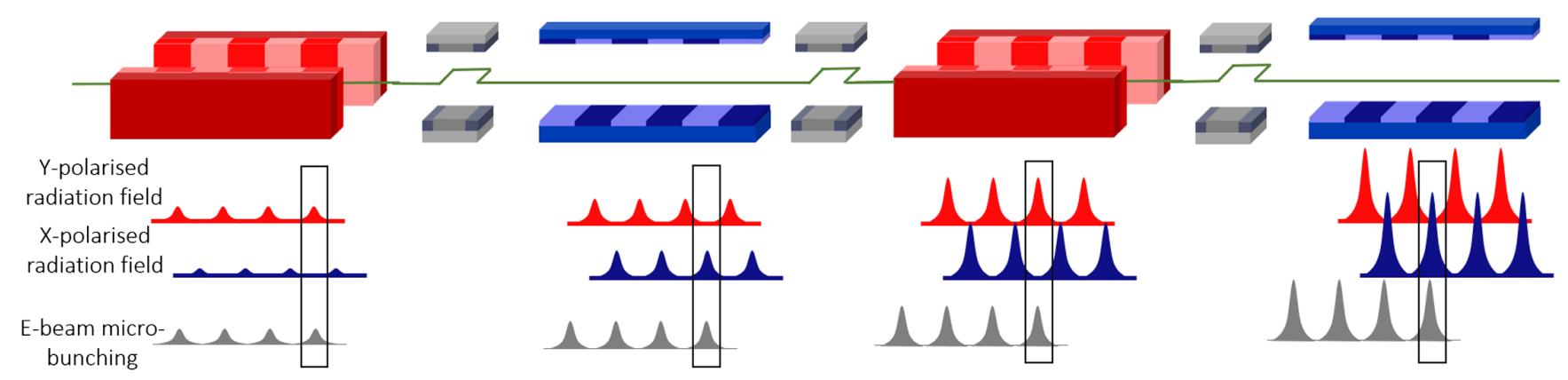

FIG. 1. Schematic layout of a section of afterburner used to generate a radiation pulse train with alternating $x$ and $y$ linear polarization. In each undulator, those regions of the electron beam with modulated microbunching emit coherently. Chicanes delay the electron beam between undulator modules so that those sections of high microbunching overlap with the appropriately polarized pulse for the undulator in which they are propagating. 
combined pulse train consists of a series of alternate, orthogonally polarized pulses.

Mode-locking in a FEL, first proposed in [21] and compacted into a mode-locked afterburner configuration in [22], creates trains of short radiation pulses via a process analogous with mode-locking in conventional cavity lasers [24]. In the mode-locked afterburner, normal FEL amplification occurs first in an electron beam prepared with an energy modulation, $\gamma(t)=\gamma_{0}+\gamma_{m} \cos \left(\omega_{m} t\right)$. This generates a periodic microbunching structure in the electron beam at the energy modulation period by creating higher FEL gain at the minima of the energy modulated beam. Chicane delays between the short undulator sections in the afterburner then map the electron beam microbunching comb onto the radiation modal pulses.

Here, a similar mapping of the microbunched comb to the mode-locked radiation generated in the orthogonally polarized afterburner modules is used to generate alternately polarized pulse trains. Figure 1 shows a schematic of how these pulse trains are generated in a planar undulator afterburner. Chicanes placed between undulator modules are chosen to delay the high microbunched regions of the electron beam to the polarized radiation pulses corresponding to the similarly polarized undulator in which they interact. The orthogonally polarized radiation pulses do not interact with the electrons in this undulator module so that they simply experience free propagation. The orthogonally polarized undulators then effectively behave as additional alternate chicane delays.

The combined slippage of the electrons with respect to a radiation wavefront between undulator modules of the same polarization should therefore be the modulation period $\lambda_{m}$. The temporal separation of the pulses of radiation with the same polarization is then $T=\lambda_{m} / c$ and the relative times of these pulses are

$$
t_{1}=n T \text {. }
$$

The orthogonally polarized pulses will then have pulse peaks at relative times:

$$
t_{2}=t_{0}+t_{1}+\Delta T
$$

where $\Delta T=s / c$ is the time for the radiation to propagate the slippage length, $s=\lambda_{m} / 2$, through the electron bunch. $t_{0}$ is a constant which may shift the radiation pulse trains relative to each other. This relative shift is achieved by adjusting the slippage between consecutive orthogonally polarized undulators to be $s+c t_{0}$ and $s-c t_{0}$. Here, we chose $t_{0}=0$ representing the case where we have equal slippage between undulator modules and therefore there is equal spacing between all pulses.

\section{SIMULATIONS}

The method is modeled using the FEL simulation code PUFFIN [25] using the parameters based on the LCLS-II project at SLAC [26] as listed in Table I. Dispersion effects within the chicanes are included in the model although chicanes which reduce dispersion and dispersionless chicanes are being developed $[27,28]$.

\section{A. Alternating linear polarization}

The method is first demonstrated using an afterburner with alternating $x$ and $y$ planar undulators that will emit correspondingly linearly polarized light. An electron beam, with a Gaussian current profile, is first prepared with a sinusoidal energy modulation of period $\lambda_{m}=$ $40 \mathrm{~nm}=32 \lambda_{r}$. This is applied via the initial conditions of the beam before any FEL interaction is simulated. Start to end simulations with full modeling of the energy modulation is left for future work. However, pre-modulation at longer wavelengths is relatively straightforward and similar modulation as presented here for a nonideal electron beam has previously been demonstrated $[29,30]$.

As with the mode-locked afterburner of [22], the electron microbunching comb is then developed in a SASE FEL "preamplifier." The simulation modeled an $x$-polarized undulator similar to that found at most current FEL facilities. The power growth in this preamplifier stage is inhibited by the electron beam energy modulation. On subsequent injection into the afterburner, the power growth in the pulsed regions becomes exponential due to their overlap with the high quality electron beam regions being maintained. There is therefore much greater radiation power generated in the afterburner than in the preamplifier. The point at which the electron beam is extracted from the preamplifier-after 900 undulator periods-is chosen so that the radiation is two orders of magnitude smaller than

TABLE I. Simulation parameters.

\begin{tabular}{lc}
\hline \hline Parameter & Value \\
\hline Electron beam energy [GeV] & 4 \\
Peak current, $I_{0}[\mathrm{kA}]$ & 1 \\
rms energy spread $\sigma_{\gamma} / \gamma$ & $1.25 \times 10^{-4}$ \\
Normalized emittance [mm-mrad] & 0.45 \\
rms beam size $\sigma_{x}[\mu \mathrm{m}]$ & 26 \\
Undulator period $\lambda_{u}[\mathrm{~cm}]$ & 3.9 \\
Resonant wavelength $\lambda_{r}[\mathrm{~nm}]$ & 1.25 \\
Modulation wavelength $\lambda_{m}[\mathrm{~nm}]$ & 40.0 \\
Modulation amplitude $\gamma_{m} / \gamma_{0}$ & $1.2 \times 10^{-3}$ \\
rms undulator parameter $\bar{a}_{u}$ & 1.72 \\
$\rho$ parameter & $1.2 \times 10^{-3}$ \\
Afterburner & 8 \\
Number of undulator periods per module & 10 \\
Chicane Delays [nm] & 36 \\
Number of undulator modules & \\
\hline \hline
\end{tabular}


the final saturated radiation power in the following afterburner.

Both the $x$ and orthogonal $y$ polarized undulator modules in the afterburner are 8 periods long, each separated by a chicane that delays the electrons by a further 8 resonant wavelengths. The total electron delay is then $s=16 \lambda_{r}=$ $\lambda_{m} / 2$ between successive undulator modules and $\lambda_{m}$ between undulators of the same polarization. This maintains overlap between the electron microbunching comb and the alternating orthogonally polarized radiation as shown in Fig. 1, leading to the amplification of radiation spikes. The orthogonal radiation spikes so generated should not interfere with each other due to their orthogonal polarization. However, as both fields are emitted by the same electron beam source, which sees only small changes between undulator modules, fluctuations in the power of one pulse train envelope should be similar to its orthogonal counterpart.

Figure 2 shows a section of the radiation power profiles and spectrum of the $x$ and $y$ polarized fields after 36 afterburner undulator modules (16 of each polarization). The additional slippage between undulator modules leads to a frequency spectrum that is broader than typical FEL output and discretized into frequency modes with modes spacing, $\Delta \omega_{s}$, as determined by the time taken for the radiation to travel the total slippage length between the same polarized undulators. The radiation pulse peaks arise from the constructive interference between the frequency modes whose phase has been fixed by the modulation, $\Delta \omega_{m}=\Delta \omega_{s}$. This is the principle of mode-locking as described in [21,24].
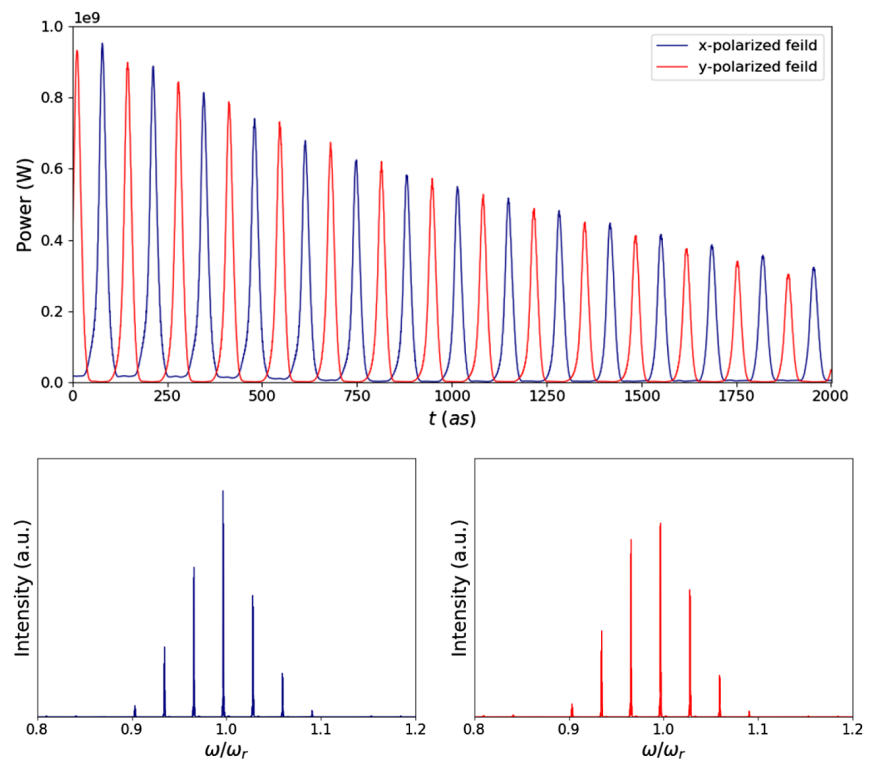

FIG. 2. Top: power vs relative time $t$ for the $x$ and $y$ polarized fields and Bottom: the corresponding spectra after 36 undulatorchicane modules.

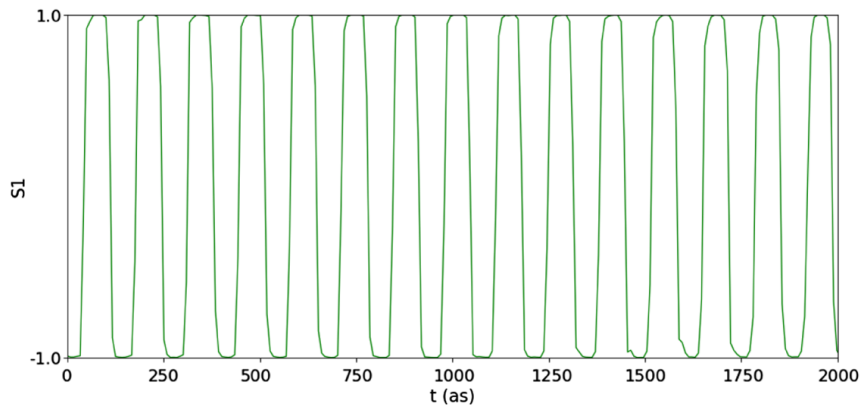

FIG. 3. The on-axis normalized Stokes parameter $s_{1}$ as a function of relative time $t$ after 36 undulator-chicane modules. It is seen that $s_{1}$ flips between positive and negative values with extremes at $\left|s_{1}\right| \approx 1.0$, indicating high degree of polarization modulation.

As the undulator modules have equal lengths, both the $x$ and $y$ polarized fields have approximately the same pulse FWHM duration of $\tau_{p} \approx 19$ as and with peak powers of $P_{p k} \approx 1 \mathrm{GW}$. The separation between each pulse is approximately 67 as corresponding to a polarization switching rate of $15 \mathrm{PHz}$.

A normalized Stokes parameter, $s_{1}$, is used to examine the degree of linear polarization in the pulses, where: $s_{1}=$ $\left(\left|E_{x}\right|^{2}-\left|E_{y}\right|^{2}\right) /\left(\left|E_{x}\right|^{2}+\left|E_{y}\right|^{2}\right)$ is the intensity difference between the $x$ and $y$ polarized fields normalized to the total intensity of the field. Values of $s_{1}= \pm 1.0$ then indicate fully linear $x$ or $y$ polarization, respectively. This is plotted as a function of time in Fig. 3 where it is seen that the polarization is highly modulated, flipping between the two polarization states. The high degree of polarization contrast is seen at the peak powers, $\left|s_{1}\right| \approx 1.0$. Across the full pulse train, $\left|s_{1}\right|>0.95$ at the peak powers, demonstrating a high degree of polarization modulation.

\section{B. Alternating circular polarization}

Pulses with polarization alternating between left and right-hand circular polarization have also been modeled. The amplifier section, which pre-bunches the electrons using SASE, remains an ( $x$-polarized) planar undulator similar to that above. The afterburner now consists of orthogonal left and right circularly polarized helical undulators.

Figure 4 shows the power profiles for the left-hand circular, LCP, and right-hand circular, RCP, polarization. The pulses now alternate between orthogonal circular polarization with the same FWHM pulse duration $\tau_{p}$ and rate as the linearly polarized case above. The stokes parameter, $s_{3}=\left(\left|E_{R}\right|^{2}-\left|E_{L}\right|^{2}\right) /\left(\left|E_{R}\right|^{2}+\left|E_{L}\right|^{2}\right)$, which gives the degree of circular polarization is plotted in Fig. 5. Across the pulse peaks, there is a high degree of circular polarization, $\left|s_{3}\right|>0.9$. This is very promising as many ultrafast polarization switching techniques cannot achieve full-handedness reversal. 


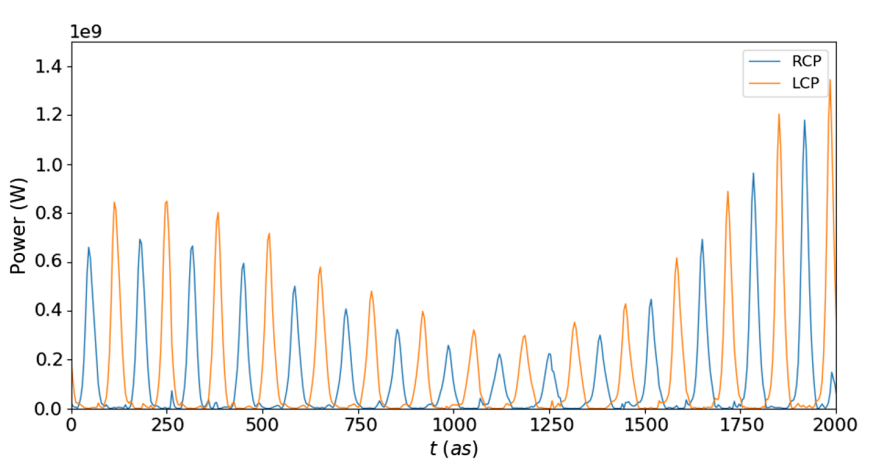

FIG. 4. Power spectrum for left and right hand polarization vs relative time at the end an afterburner with alternating polarized helical undulator modules.

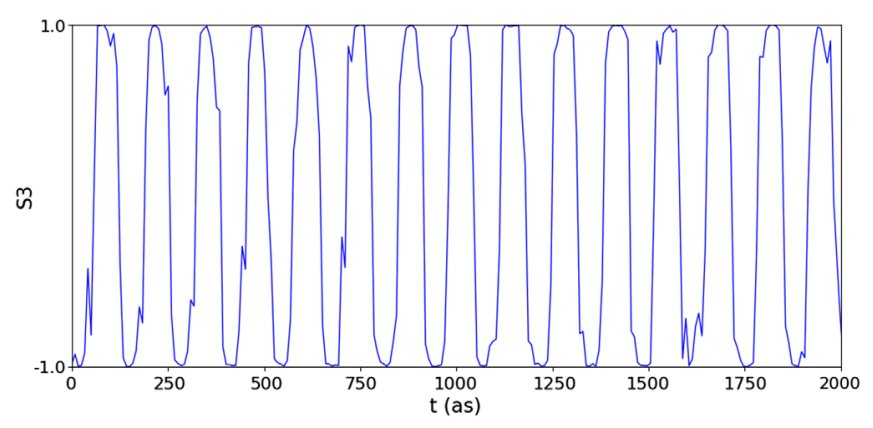

FIG. 5. The on-axis normalized Stokes parameter $s_{3}$ as a function of relative time $t$ after 36 undulator-chicane modules. It is seen that $s_{3}$ flips between positive and negative values with extremes at $\left|s_{3}\right| \approx 1.0$, indicating high degree of polarization modulation.

\section{CONCLUSIONS}

This paper demonstrates a novel method to generate attosecond polarization modulation in a short wavelength FEL without the need for any optical components. This represents a considerable improvement in wavelength and timescales over any other methods currently available, and could be expected to drive forward new experimental opportunities in fundamental science. The simulation parameters used considered soft $\mathrm{x}$-ray pulses similar to the LCLS-II, however, this is by no means the limit of the wavelengths available with this set up. Extrapolating from the simulations of a hard $\mathrm{x}$-ray mode-locked afterburner as presented in [22], the same parameters adapted to generate alternating polarized pulses here would generate pulse separation times of 5 as, approximately one fifth of the atomic unit of time. Discussion of scaling the mode-locked afterburner to yet higher photon energies provided in [22] should also apply to the methods described here. Given the broad scaling of FEL wavelength operation, the method described will also be applicable to longer wavelengths, again opening up new experimental opportunities.

As well as operating across a broad range of wavelengths, the method could be adapted to meet other specific experimental requirements. The temporal shift between pulse trains of orthogonal polarizations may be controlled to bring alternating pulses close together followed by a longer time interval. The time duration of the different pulse types may also be altered by the length of the different types of undulators to generate pulse trains with a pulse of one polarization followed by a shorter pulse with the orthogonal polarization. However, it is noted that this will also result in different pulse powers and bandwidths which would need further consideration.

This method also provides a promising broader avenue to tailor FEL output and provide bespoke radiation for experiments. Further development of the method will include alternating other pulse properties such as the wavelength, e.g., using the work of [31], or orbital angular momentum of the pulses [32]. While experimental implementation of mode-locking has not yet been trialed, it may be advantageous to consider alternating pulse structure capabilities when upgrading FELs to include mode-locking.

\section{ACKNOWLEDGMENTS}

We are grateful to funding from the Science and Technology Facilities Council (Agreement Number 4163192 Release\#3); ARCHIEWeSt HPC, EPSRC Grant No. EP/K000586/1; EPSRC Grant No. EP/ M011607/1; John von Neumann Institute for Computing (NIC) on JUROPA at Jülich Supercomputing Centre (JSC), Project No. HHH20.

[1] N. Berova, P. L. Polavarapu, K. Nakanishi, and R. W. Woody, Comprehensive Chiroptical Spectroscopy: Applications in Stereochemical Analysis of Synthetic Compounds, Natural Products, and Biomolecules (John Wiley \& Sons, New York, 2012), Vol. 2.

[2] U. Hergenhahn, E. E. Rennie, O. Kugeler, S. Marburger, T. Lischke, I. Powis, and G. Garcia, Photoelectron circular dichroism in core level ionization of randomly oriented pure enantiomers of the chiral molecule camphor, J. Chem. Phys. 120, 4553 (2004).

[3] A. Kirilyuk, A. V. Kimel, and T. Rasing, Ultrafast optical manipulation of magnetic order, Rev. Mod. Phys. 82, 2731 (2010).

[4] H. Rubinsztein-Dunlop et al., Roadmap on structured light, J. Opt. 19, 013001 (2017).

[5] S. Rozen, A. Comby, E. Bloch, S. Beauvarlet, D. Descamps, B. Fabre, S. Petit, V. Blanchet, B. Pons, N. Dudovich, and Y. Mairesse, Controlling Subcycle Optical Chirality in the Photoionization of Chiral Molecules, Phys. Rev. X 9, 031004 (2019).

[6] G. Schütz, W. Wagner, W. Wilhelm, P. Kienle, R. Zeller, R. Frahm, and G. Materlik, Absorption of Circularly Polarized X-rays in Iron, Phys. Rev. Lett. 58, 737 (1987).

[7] G. van der Laan and A. I. Figueroa, X-ray magnetic circular dichroisma versatile tool to study magnetism, Coord. Chem. Rev. 277-278, 95 (2014), following Chemical Structures using Synchrotron Radiation. 
[8] M. Suzuki, N. Kawamura, and T. Ishikawa, Application of optical scanner to switching of x-ray photon helicities at khz range, Rev. Sci. Instrum. 74, 19 (2003).

[9] M. Först, C. Manzoni, S. Kaiser, Y. Tomioka, Y. Tokura, R. Merlin, and A. Cavalleri, Nonlinear phononics as an ultrafast route to lattice control, Nat. Phys. 7, 854 (2011).

[10] T. Kampfrath, A. Sell, G. Klatt, A. Pashkin, S. Mährlein, T. Dekorsy, M. Wolf, M. Fiebig, A. Leitenstorfer, and R. Huber, Coherent terahertz control of antiferromagnetic spin waves, Nat. Photonics 5, 31 (2011).

[11] J. Sánchez-Barriga, E. Golias, A. Varykhalov, J. Braun, L. V. Yashina, R. Schumann, J. Minár, H. Ebert, O. Kornilov, and O. Rader, Ultrafast spin-polarization control of dirac fermions in topological insulators, Phys. Rev. B 93, 155426 (2016).

[12] J. D. Bull, N. A. Jaeger, H. Kato, M. Fairburn, A. Reid, and P. Ghanipour, 40-GHz electro-optic polarization modulator for fiber optic communications systems, in Photonics North 2004: Optical Components and Devices, Vol. 5577, edited by J. C. Armitage, S. Fafard, R. A. Lessard, and G. A. Lampropoulos, International Society for Optics and Photonics (SPIE, New York, 2004), pp. 133-143.

[13] Y. Yang, K. Kelley, E. Sachet, S. Campione, T. S. Luk, J.-P. Maria, M. B. Sinclair, and I. Brener, Femtosecond optical polarization switching using a cadmium oxide-based perfect absorber, Nat. Photonics 11, 390 (2017).

[14] L. H. Nicholls, F. J. Rodríguez-Fortuño, M. E. Nasir, R. M. Córdova-Castro, N. Olivier, G. A. Wurtz, and A. V. Zayats, Ultrafast synthesis and switching of light polarization in nonlinear anisotropic metamaterials, Nat. Photonics 11, 628 (2017).

[15] K. Holldack, C. Schüssler-Langeheine, P. Goslawski, N. Pontius, T. Kachel, F. Armborst, M. Ries, A. Schälicke, M. Scheer, W. Frentrup et al., Flipping the helicity of x-rays from an undulator at unprecedented speed, Commun. Phys. 3, 61 (2020).

[16] S. Serkez, A. Trebushinin, M. Veremchuk, and G. Geloni, Method for polarization shaping at free-electron lasers, Phys. Rev. Accel. Beams 22, 110705 (2019).

[17] A. A. Lutman, T. J. Maxwell, J. P. MacArthur, M. W. Guetg, N. Berrah, R. N. Coffee, Y. Ding, Z. Huang, A. Marinelli, S. Moeller et al., Fresh-slice multicolour x-ray free-electron lasers, Nat. Photonics 10, 745 (2016).

[18] B. W. McNeil and N. R. Thompson, X-ray free-electron lasers, Nat. Photonics 4, 814 (2010).

[19] R. Bonifacio, L. De Salvo, P. Pierini, N. Piovella, and C. Pellegrini, Spectrum, Temporal Structure, and Fluctuations in a High-Gain Free-Electron Laser Starting from Noise, Phys. Rev. Lett. 73, 70 (1994).

[20] A. A. Lutman, J. P. MacArthur, M. Ilchen, A. O. Lindahl, J. Buck, R. N. Coffee, G. L. Dakovski, L. Dammann, Y. Ding, H. A. Dürr et al., Polarization control in an X-ray free-electron laser, Nat. Photonics 10, 468 (2016).

[21] N. R. Thompson and B. W. J. McNeil, Mode Locking in a Free-Electron Laser Amplifier, Phys. Rev. Lett. 100, 203901 (2008).

[22] D. J. Dunning, B. W. J. McNeil, and N. R. Thompson, Few-Cycle Pulse Generation in an X-ray Free-Electron Laser, Phys. Rev. Lett. 110, 104801 (2013).

[23] E. Hemsing, Coherent photons with angular momentum in a helical afterburner, Phys. Rev. Accel. Beams 23, 020703 (2020).

[24] A. E. Siegman, Lasers (University Science Books, Mill Valley, CA, 1986), Vol. 37, p. 169.

[25] L. Campbell and B. McNeil, Puffin: A three dimensional, unaveraged free electron laser simulation code, Phys. Plasmas 19, 093119 (2012).

[26] R. W. Schoenlein, New Science Opportunities Enabled by LCLS-II X-ray Lasers, SLAC National Accelerator Laboratory Tech. Report No. SLAC-R-1053, 2015.

[27] J. Clarke, J. Jones, and N. Thompson, A compact, modular electron beam delay line for use in novel free-electron laser schemes, Conf. Proc. C 1205201, 1759 (2012).

[28] N. Thompson, XFEL isochronous chicanes: Feasibility study, in Proc. FEL'19, Free Electron Laser Conference No. 39 (JACoW Publishing, Geneva, Switzerland, 2019), pp. 658-660.

[29] D. Dunning, N. Thompson, P. Williams, and B. McNeil, Start-to-end simulations of SASE and hhg-seeded modelocked FEL, in Proceedings of FEL09 Conference, Liverpool (2009), pp. 165-168.

[30] D. J. Dunning, B. W. J. Mc Neil, N. R. Thompson, and P. H. Williams, Start-to-end modelling of a mode-locked optical klystron free electron laser amplifier, Phys. Plasmas 18, 073104 (2011).

[31] L. T. Campbell, B. W. J. McNeil, and S. Reiche, Twocolour free electron laser with wide frequency separation using a single monoenergetic electron beam, New J. Phys. 16, 103019 (2014).

[32] E. Hemsing, A. Marinelli, and J. B. Rosenzweig, Generating Optical Orbital Angular Momentum in a High-Gain Free-Electron Laser at the First Harmonic, Phys. Rev. Lett. 106, 164803 (2011). 